\title{
Supporting Information \\ Effects of phase state and phase separation on dimethylamine uptake of ammonium sulfate and ammonium sulfate-sucrose mixed particles
}

\author{
Wing-Sy Wong DeRieux ${ }^{1 \S}$, Pascale S. J. Lakey ${ }^{1 \S}$, Yangxi Chu ${ }^{2}$, Chak K. Chan ${ }^{3}$, \\ Hayley S. Glicker ${ }^{1}$, James N. Smith ${ }^{1}$, Andreas Zuend ${ }^{4}$, and Manabu Shiraiwa ${ }^{1^{*}}$ \\ [1] Department of Chemistry, University of California, Irvine, CA 92697-2025, USA \\ [2] Chinese Research Academy of Environmental Sciences, Beijing 100012, China \\ [3] School of Energy and Environment, City University of Hong Kong, Hong Kong, China \\ [4] Department of Atmospheric and Oceanic Sciences, McGill University, Montreal, QC H3A \\ 0B9, Canada \\ $\S$ These authors contributed equally to this work. \\ *Correspondence to: M. Shiraiwa (m.shiraiwa@uci.edu)
}



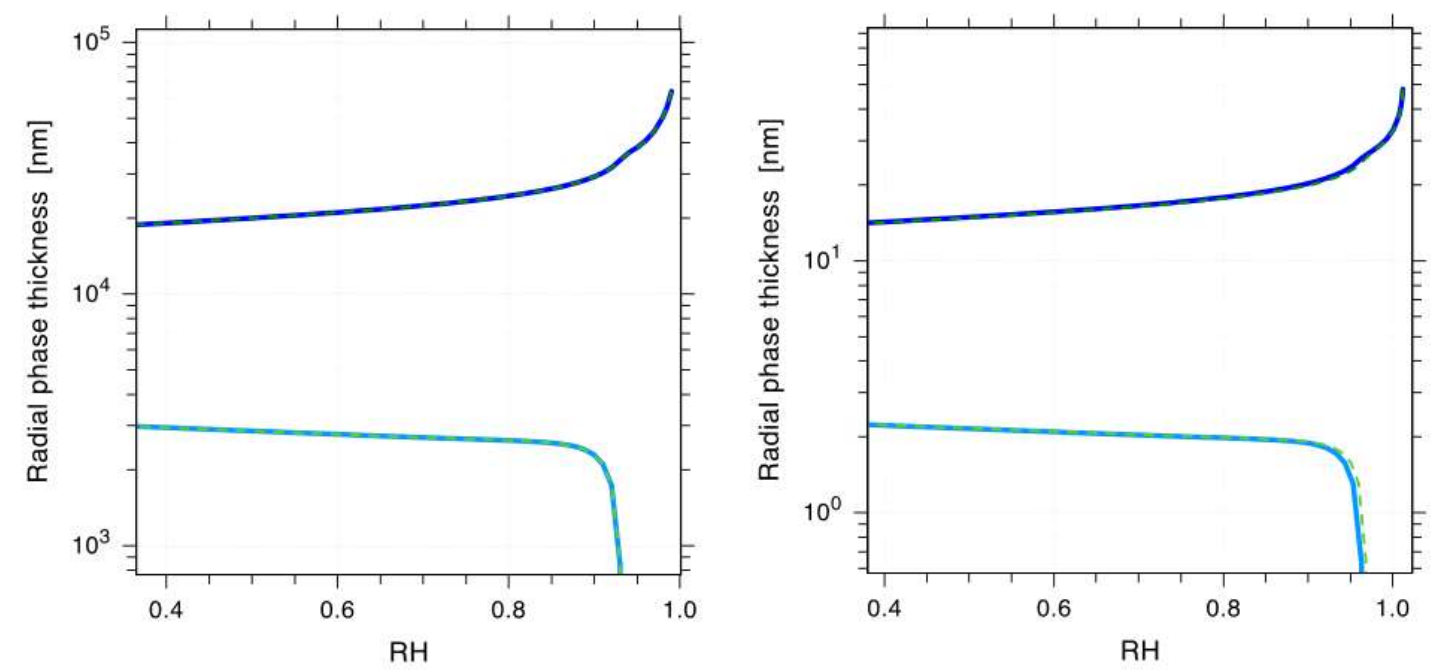

Figure S1. Dependence of phase thickness on RH for (a) a $40 \mu \mathrm{m}$ dry diameter particle and; (b) a $30 \mathrm{~nm}$ dry diameter particle for the dehydration branch (i.e. without a solid phase) at $T=297 \mathrm{~K}$. The dark blue solid line is the AS-rich $(\alpha)$ phase (the particle core) and the light blue solid line is the sucrose-rich $(\beta)$ phase (the particle shell). The blue lines represent a surface tension of 44-72 $\mathrm{mN} \mathrm{m}^{-1}$ while the dashed green lines represent the case with a constant surface tension of $73 \mathrm{mN}$ $\mathrm{m}^{-1}$.
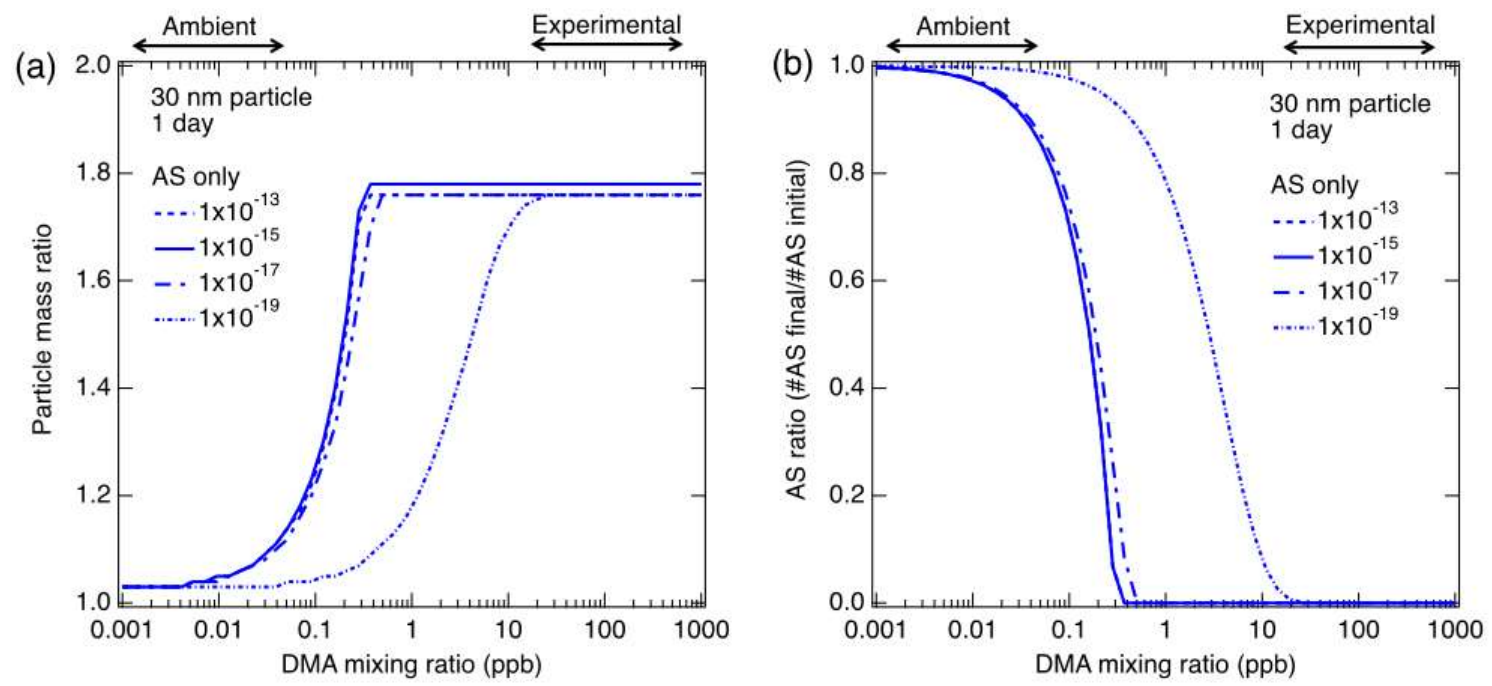

Figure S2. Sensitivity of (a) predicted particle mass ratios (particle mass after exposure to DMA divided by initial particle mass) and (b) AS ratio (number of AS after DMA exposure divided by initial number of $\mathrm{AS}$ ) to variation in the reaction rate coefficient at $\mathrm{RH}=30 \%$. Kinetic modeling was used to simulate DMA uptake by pure AS particles with $30 \mathrm{~nm}$ diameter over 1 day at varying DMA mixing ratios. DMA mixing ratios range from ppt levels found in the atmosphere to ppm levels used in experimental data. 

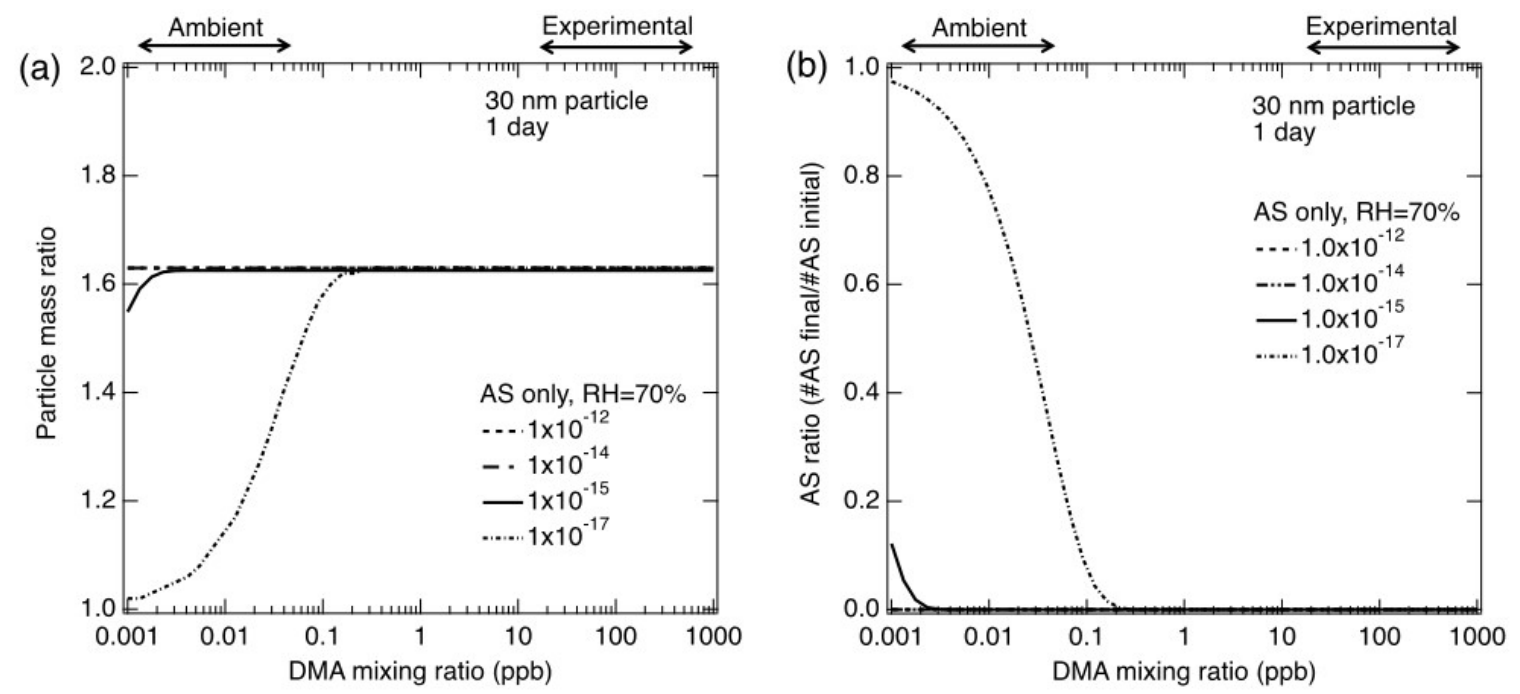

Figure S3. Sensitivity of (a) predicted particle mass ratios (particle mass after exposure to DMA divided by initial particle mass) and (b) AS ratio (number of AS after DMA exposure divided by initial number of AS) to variation in the reaction rate coefficient at $\mathrm{RH}=70 \%$. Kinetic modeling was used to simulate DMA uptake by pure AS particles with $30 \mathrm{~nm}$ diameter over 1 day at varying DMA mixing ratios. DMA mixing ratios range from ppt levels found in the atmosphere to ppm levels used in experimental data.
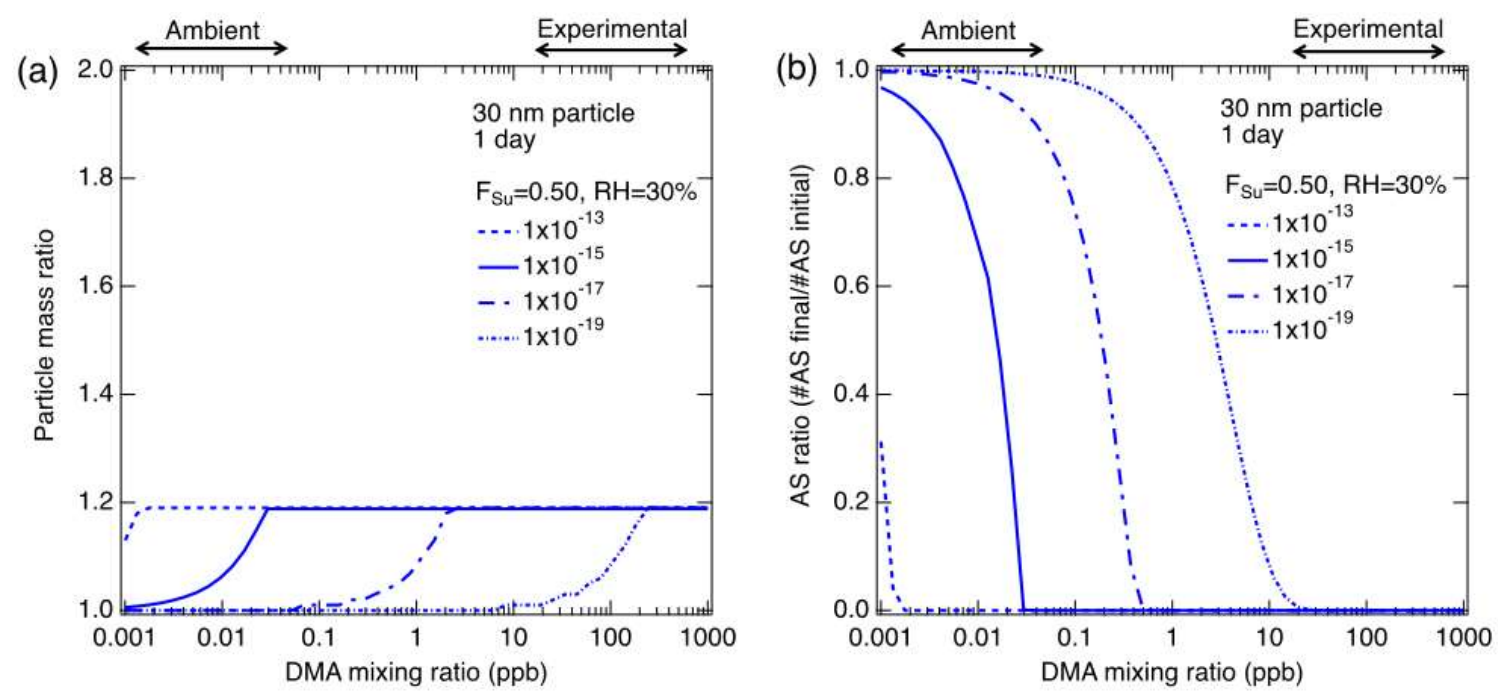

Figure S4. Sensitivity of (a) predicted particle mass ratios (particle mass after exposure to DMA divided by initial particle mass) and (b) AS ratio (number of AS after DMA exposure divided by initial number of AS) to variation in the reaction rate coefficient at $\mathrm{RH}=30 \%$. Kinetic modeling was used to simulate DMA uptake by AS-sucrose $\left(F_{\text {su }}=0.50\right)$ particles with $30 \mathrm{~nm}$ diameter over 1 day at varying DMA mixing ratios. DMA mixing ratios range from ppt levels found in the atmosphere to ppm levels used in experimental data. 

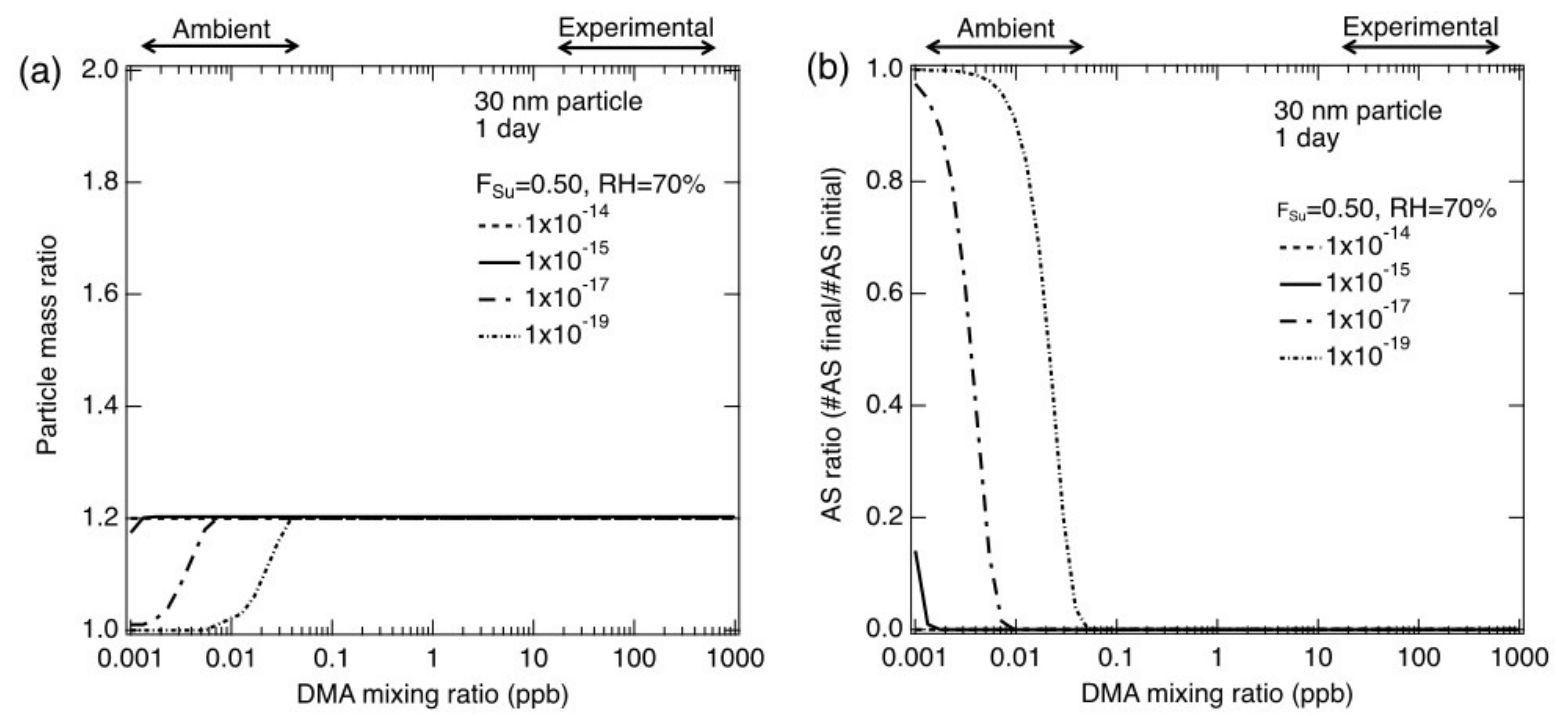

Figure S5. Sensitivity of (a) predicted particle mass ratios (particle mass after exposure to DMA divided by initial particle mass) and (b) AS ratio (number of AS after DMA exposure divided by initial number of AS) to variation in the reaction rate coefficient at $\mathrm{RH}=70 \%$. Kinetic modeling was used to simulate DMA uptake by AS-sucrose $\left(F_{\text {su }}=0.50\right)$ particles with $30 \mathrm{~nm}$ diameter over 1 day at varying DMA mixing ratios. DMA mixing ratios range from ppt levels found in the atmosphere to ppm levels used in experimental data. 
Table S1. General parameters used for KM-GAP. Values are for all species unless otherwise indicated.

\begin{tabular}{|c|c|c|c|c|c|}
\hline parameter & description & units & value & range & source \\
\hline$D_{\mathrm{g}}$ & $\begin{array}{l}\text { Gas diffusion } \\
\text { coefficient }\end{array}$ & $\mathrm{cm}^{2} \mathrm{~s}^{-1}$ & 0.1 & - & $\begin{array}{l}\text { EPA tools for } \\
\text { online site } \\
\text { assessment }\end{array}$ \\
\hline$\alpha_{0}$ & $\begin{array}{c}\text { Surface } \\
\text { accommodation } \\
\text { coefficient on free } \\
\text { substrate } \\
\end{array}$ & - & 1 & - & $\begin{array}{c}\text { Shiraiwa et al., } \\
\text { Nat. Chem., 3, } \\
291 \text { (2011) }\end{array}$ \\
\hline$\tau_{\mathrm{d}}$ & $\begin{array}{l}\text { Desorption } \\
\text { lifetime }\end{array}$ & $\mathrm{s}$ & $\begin{array}{l}(\mathrm{DMA}) 1.0 \times 10^{-9} \\
\left(\mathrm{NH}_{3}\right) \text { insensitive }\end{array}$ & - & $\begin{array}{l}\text { Shiraiwa et al., } \\
\text { Nat. Chem., 3, } \\
291 \text { (2011) }\end{array}$ \\
\hline$k_{\mathrm{ssl}}$ and $k_{\mathrm{br}}$ & $\begin{array}{l}\text { reaction rate } \\
\text { constant for } \\
\text { reaction (1) }\end{array}$ & $\mathrm{cm}^{3} \mathrm{~s}^{-1}$ & $1.0 \times 10^{-15}$ & $\begin{array}{l}1.0 \times 10^{-10}- \\
1.0 \times 10^{-17}\end{array}$ & $\begin{array}{c}\text { fitting } \\
\text { parameter }\end{array}$ \\
\hline$H$ & $\begin{array}{l}\text { Henry's law } \\
\text { coefficient }\end{array}$ & $\begin{array}{l}\mathrm{mol} \mathrm{cm}^{3} \\
\mathrm{~atm}^{-1}\end{array}$ & $5.0 \times 10^{-2}$ & $\begin{array}{l}4.75 \times 10^{-2}- \\
5.25 \times 10^{-2}\end{array}$ & $\begin{array}{l}\text { Sander, Atmos. } \\
\text { Chem. Phys., } \\
\text { 15, } 4399 \text { (2015) }\end{array}$ \\
\hline
\end{tabular}

Table S2. Input parameters for ammonium sulfate only particles.

\begin{tabular}{|r|c|c|}
\hline & $\begin{array}{c}\text { Initial dry } \\
\text { radius (cm) }\end{array}$ & $\begin{array}{c}\text { Bulk diffusion } \\
\text { coeff }\left(\mathrm{cm}^{2} \mathrm{~s}^{-1}\right)\end{array}$ \\
\hline $\begin{array}{c}\text { Fsu=0.00 (AS } \\
\text { only) }\end{array}$ & & \\
\hline $1 \%$ & $2.8 \times 10^{-3}$ & $1.25 \times 10^{-11}$ \\
\hline $10 \%$ & $2.35 \times 10^{-3}$ & $6.25 \times 10^{-11}$ \\
\hline $20 \%$ & $2.35 \times 10^{-3}$ & $1.5 \times 10^{-10}$ \\
\hline $30 \%$ & $2.0 \times 10^{-3}$ & $1.5 \times 10^{-10}$ \\
\hline $70 \%$ & $2.45 \times 10^{-3}$ & $5.0 \times 10^{-6}$ \\
\hline
\end{tabular}


Table S3. Input parameters for AS-sucrose mixed particles.

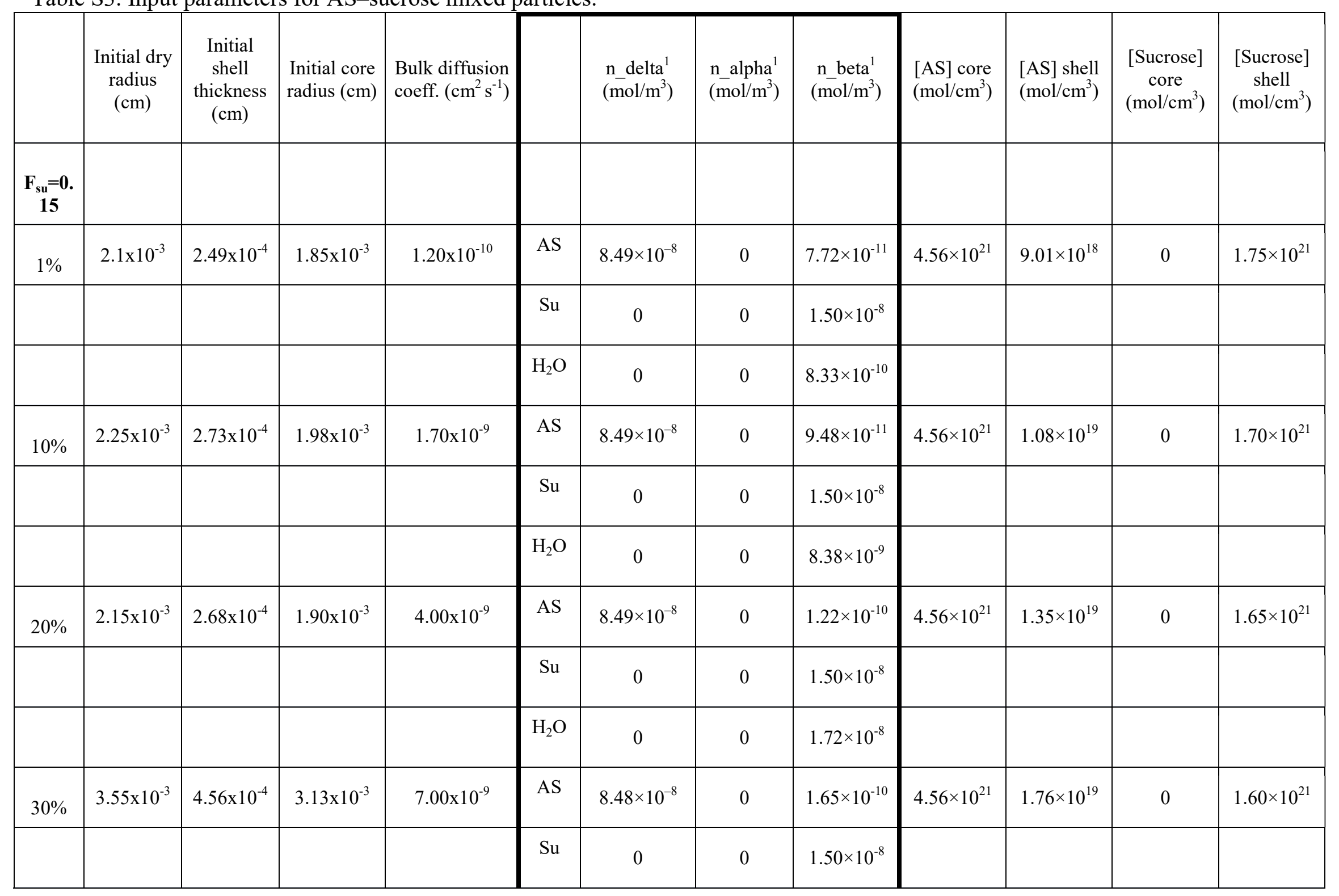




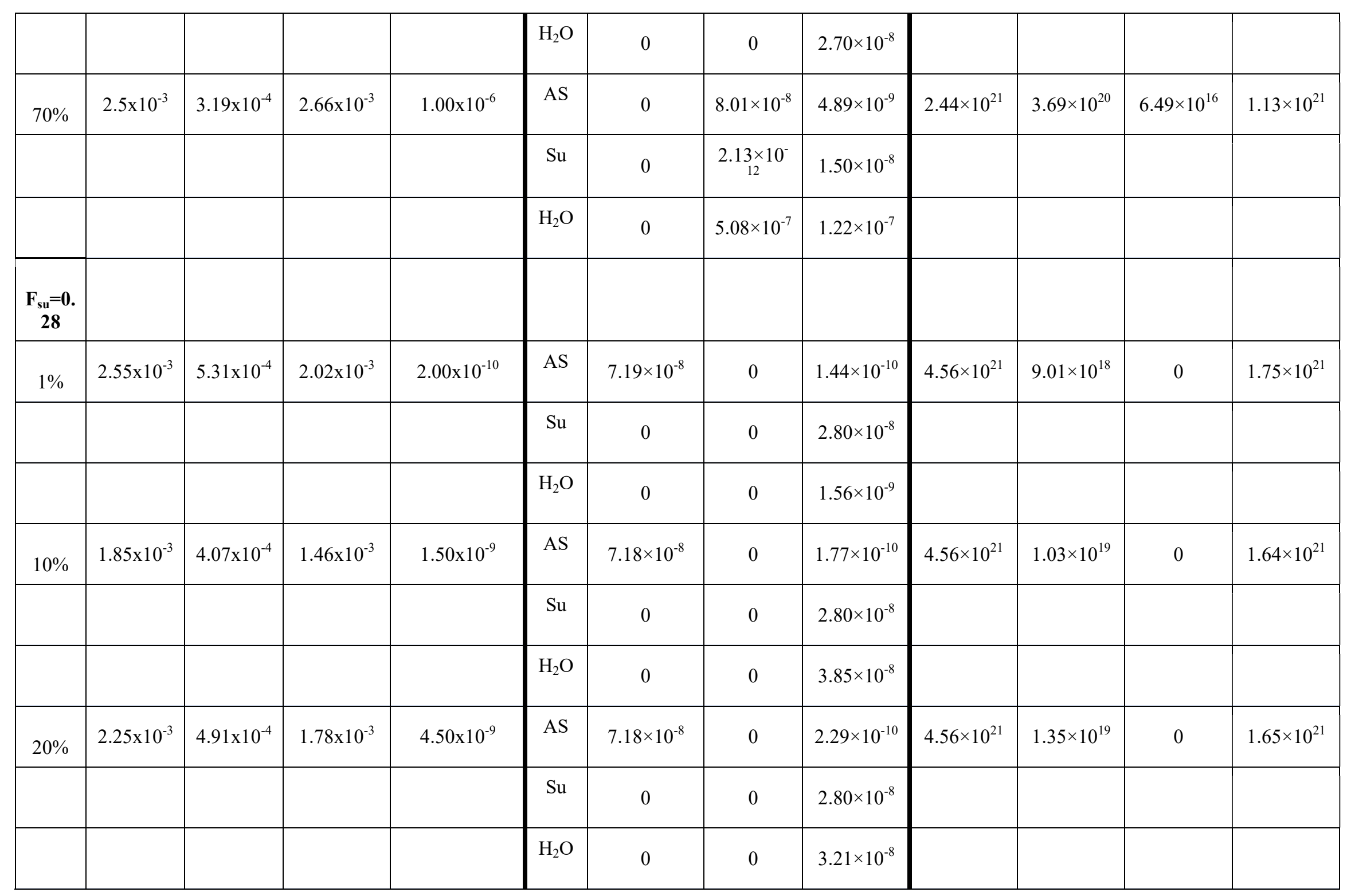




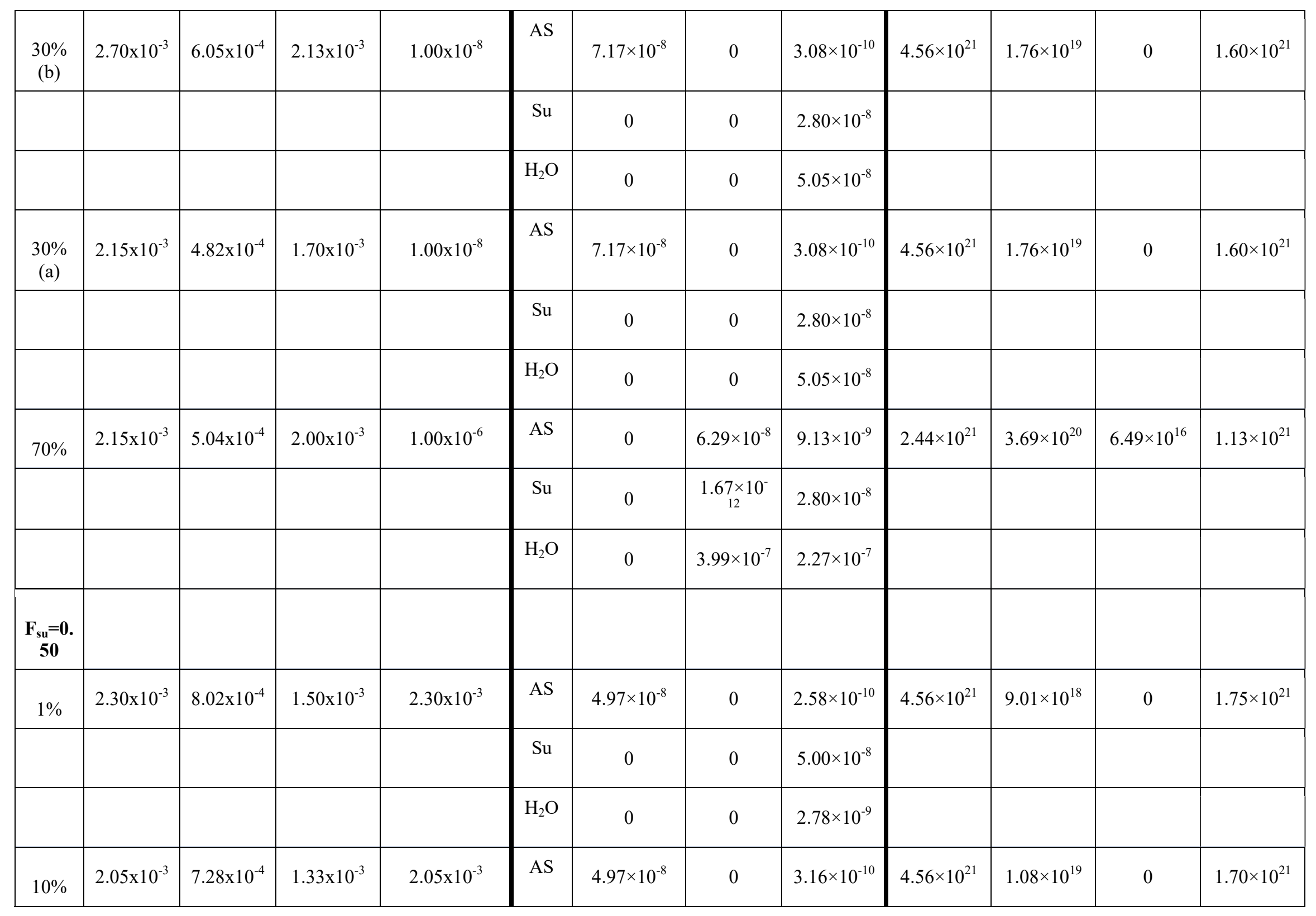




\begin{tabular}{|c|c|c|c|c|c|c|c|c|c|c|c|c|}
\hline & & & & & $\mathrm{Su}$ & 0 & 0 & $5.00 \times 10^{-8}$ & & & & \\
\hline & & & & & $\mathrm{H}_{2} \mathrm{O}$ & 0 & 0 & $2.79 \times 10^{-8}$ & & & & \\
\hline \multirow[t]{2}{*}{$20 \%$} & $1.85 \times 10^{-3}$ & $6.72 \times 10^{-4}$ & $1.20 \times 10^{-3}$ & $1.85 \times 10^{-3}$ & AS & $4.96 \times 10^{-8}$ & 0 & $4.08 \times 10^{-10}$ & $4.56 \times 10^{21}$ & $1.35 \times 10^{19}$ & 0 & $1.65 \times 10^{21}$ \\
\hline & & & & & $\mathrm{Su}$ & 0 & 0 & $5.00 \times 10^{-8}$ & & & & \\
\hline \multirow[t]{2}{*}{$30 \%$} & $2.05 \times 10^{-3}$ & $7.62 \times 10^{-4}$ & $1.33 \times 10^{-3}$ & $2.05 \times 10^{-3}$ & AS & $4.95 \times 10^{-8}$ & 0 & $5.49 \times 10^{-10}$ & $4.56 \times 10^{21}$ & $1.76 \times 10^{19}$ & 0 & $1.60 \times 10^{21}$ \\
\hline & & & & & $\mathrm{Su}$ & 0 & 0 & $5.00 \times 10^{-8}$ & & & & \\
\hline \multirow{2}{*}{$70 \%$} & & & & & $\mathrm{Su}$ & 0 & $\underset{13}{8.94 \times 10^{-}}$ & $5.00 \times 10^{-8}$ & & & & \\
\hline & & & & & $\mathrm{H}_{2} \mathrm{O}$ & 0 & $2.14 \times 10^{-7}$ & $4.06 \times 10^{-7}$ & & & & \\
\hline $\begin{array}{c}\text { Sourc } \\
\mathrm{e}\end{array}$ & $\begin{array}{c}\text { Chu \& } \\
\text { Chan, J } \\
\text { PhysChe } \\
\text { m A, 121, } \\
206 \\
(2017)\end{array}$ & KM-GAP & KM-GAP & $\begin{array}{c}\text { fitting } \\
\text { parameter }\end{array}$ & & AIOMFAC & AIOMFAC & AIOMFAC & AIOMFAC & AIOMFAC & AIOMFAC & AIOMFAC \\
\hline
\end{tabular}


${ }^{1}$ Initial moles of indicated component in the indicated phase before DMA uptake predicted by the AIOMFAC-LLE model. Delta phase corresponds to the solid phase in the hydration case. Beta phase and alpha phase correspond to the sucrose-rich and AS-rich phases, respectively, that are predicted to form in both the hydration and dehydration cases by the thermodynamic model. For further information, please refer to the main article text. 